\title{
Increased functional connectivity in intrinsic neural networks in individuals with aniridia
}

\section{Jordan E. Pierce ${ }^{1}$, Cynthia E. Krafft ${ }^{1}$, Amanda L. Rodrigue ${ }^{1}$, Anastasia M. Bobilev ${ }^{2}$, James D. Lauderdale ${ }^{2,3}$ and Jennifer E. McDowell ${ }^{1,2}$ *}

\author{
${ }^{1}$ Department of Psychology, University of Georgia, Athens, GA, USA \\ ${ }^{2}$ Department of Neuroscience, University of Georgia, Athens, GA, USA \\ ${ }^{3}$ Department of Cellular Biology, University of Georgia, Athens, GA, USA
}

\section{Edited by:}

Hans-Jochen Heinze, University of Magdeburg, Germany

Reviewed by:

Daniele Marinazzo, University of

Gent, Belgium

Michael B. Hoffmann,

Otto-von-Guericke University,

Germany

*Correspondence:

Jennifer E. McDowell, Department of Psychology, University of

Georgia, 125 Baldwin Street,

Athens, GA 30602, USA

e-mail: jemcd@uga.edu
Mutations affecting the PAX6 gene result in aniridia, a condition characterized by the lack of an iris and other panocular defects. Among humans with aniridia, structural abnormalities also have been reported within the brain. The current study examined the functional implications of these deficits through "resting state" or task-free functional magnetic resonance imaging (fMRI) in 12 individuals with aniridia and 12 healthy ageand gender-matched controls. Using independent components analysis (ICA) and dual regression, individual patterns of functional connectivity associated with three intrinsic connectivity networks (ICNs; executive control, primary visual, and default mode) were compared across groups. In all three analyses, the aniridia group exhibited regions of greater connectivity correlated with the network, while the controls did not show any such regions. These differences suggest that individuals with aniridia recruit additional neural regions to supplement function in critical intrinsic networks, possibly due to inherent structural or sensory abnormalities related to the disorder.

Keywords: functional connectivity, aniridia, PAX6, resting state, dual regression, fMRI

\section{INTRODUCTION}

Aniridia is a panocular disorder (for a review see Lee et al., 2008; Hingorani et al., 2012), which is characterized by the absence or hypoplasia of the iris for which it is named, lens opacification, increased intraocular pressure, corneal keratopathy, foveal hypoplasia, and optic nerve hypoplasia (Nelson et al., 1984; McCulley et al., 2005). Symptoms are present in early childhood and worsen throughout the lifespan, with many individuals eventually developing cataracts and/or glaucoma (Hingorani et al., 2009, 2012; Gramer et al., 2012). In humans the development of aniridia is linked to PAX6, a highly conserved paired-box transcription factor that controls key steps in eye development in metazoans (Halder et al., 1995; Gehring, 1996; van Heyningen and Williamson, 2002). Heterozygous null mutations within PAX6, cytogenetic deletions of chromosome 11 p13 that encompass PAX6 or chromosomal rearrangements that prevent PAX6 expression from one allele are all causal for aniridia (Ton et al., 1991; Fantes et al., 1995; Lauderdale et al., 2000; van Heyningen and Williamson, 2002; Robinson et al., 2008).

In addition to its well-known function in eye development, PAX6 plays several roles in the developing brain and spinal cord (Walther and Gruss, 1991; Osumi et al., 2008), including control of proliferation, specification, and differentiation of neural progenitor cells (Götz et al., 1998; Warren et al., 1999; Estivill-Torrus et al., 2002; Heins et al., 2002). In mice, mutations in PAX6 are associated with developmental abnormalities of the brain (Boretius et al., 2009; Tuoc et al., 2009; Georgala et al., 2011). In humans, abnormalities in brain structure have been reported for individuals with aniridia based on analyses of structural magnetic resonance images including: reduced volume of the interhemispheric commissures and corpus callosum (Sisodiya et al., 2001; Free et al., 2003; Bamiou et al., 2007), absence or hypoplasia of the pineal gland and olfactory bulb, polymicrogyria (Mitchell et al., 2003), reduced overall gray matter volume (Sisodiya et al., 2001), and reduced gray matter concentration in the cerebellum and the occipital lobes (Free et al., 2003).

In addition to structural abnormalities, basic functional deficits in vision (as described above (Lee et al., 2008)), audition (impaired interhemispheric transfer of auditory signals (Bamiou et al., 2004, 2007)), and olfaction (mild hyposmia to anosmia (Sisodiya et al., 2001)) have been reported previously. Few studies, however, have examined higher cognitive processes in this population. General cognitive functioning appears to be preserved in aniridia (Thompson et al., 2004) except in cases of Wilms tumor-aniridia-genitourinary-mental retardation (WAGR) syndrome (Schwartz et al., 1994; Fischbach et al., 2005); however, using measures of verbal executive function and social cognition, Heyman et al. (1999) reported impaired cognitive performance in a family with aniridia compared to healthy controls. These same individuals later participated in the only previous functional magnetic resonance imaging (fMRI) study published to date on humans with aniridia (Ellison-Wright et al., 2004). 
Seven affected family members were tested on verbal fluency and response inhibition tasks. The subjects with aniridia exhibited less activation of fronto-striatal-thalamic circuitry than controls during the task. Additionally, structural abnormalities including excess gray matter and reduced white matter were observed that suggested a potential link between the underlying neural architecture, cognitive performance, and patterns of functional activation.

The sensory deficits associated with the population of interest present challenges in using traditional fMRI stimuli to evaluate cognitive and sensory processing. One means of overcoming this obstacle is to implement task-free imaging techniques to measure intrinsic neural activation patterns while the participant is in a relaxed, awake condition commonly known as "resting-state" (Beckmann et al., 2005; Fox et al., 2005; Damoiseaux et al., 2006). When a participant has no specific task to complete, thoughts are free to wander and cognition may periodically become focused within certain intrinsic domains. Accordingly, widespread neural networks that regularly function together during active behaviors will spontaneously co-activate at low frequencies during a task-free fMRI session. These patterns of activity can be measured via the blood oxygenation level dependent (BOLD) signal, and inter-correlated to determine the intrinsic functional connectivity of various regions across the cortex (Biswal et al., 1995; Greicius et al., 2003; Damoiseaux et al., 2006; De Luca et al., 2006; Fox and Raichle, 2007; Rogers et al., 2007). Furthermore, this inherent neural synchrony has been related to activity during cognitive tasks (Seeley et al., 2007; Sharp et al., 2011; Kannurpatti et al., 2012; Sala-Llonch et al., 2012) and, thus, can provide insight into cognitive function without the need for stimulus presentation, task compliance and/or overt behavioral measures.

In the present study three well-documented intrinsic connectivity networks (ICNs; Seeley et al., 2007) were investigated: the executive control network, the primary visual network, and the default mode network. The executive control network (Greicius et al., 2003; Beckmann et al., 2005; Damoiseaux et al., 2006; Seeley et al., 2007), based in frontal cortex, relates to cognitive control processes and mediates activity in other brain regions; it was chosen due to previously reported differences in verbal executive control and frontal activation associated with aniridia (Ellison-Wright et al., 2004). The primary visual ICN includes neurons that process visual sensory input in its early stages and has been shown to be disrupted in individuals with visual deficits (Liu et al., 2007; Yu et al., 2008; Qin et al., 2013). Finally, the default mode network (Raichle et al., 2001) represents ongoing neural processes including self-referential thought (Gusnard et al., 2001; Fransson, 2005; Kim, 2010) and memory retrieval (Greicius et al., 2003; Buckner et al., 2008), and is often found to be anti-correlated with brain activity during demanding cognitive tasks (Fox et al., 2005). It was hypothesized that the functional connectivity for the aniridia group would be reduced in all ICNs relative to the comparison group due to their altered sensory inputs, neural development, and structural organization. Characterizing the synchrony of neural networks during task-free fMRI in individuals with aniridia, in conjunction with previously identified structural abnormalities in this population, can elucidate differences in neural function that result from the disruption of the PAX6 protein in this condition.

\section{MATERIALS AND METHODS PARTICIPANTS AND PROCEDURE}

Fourteen individuals with aniridia and 15 healthy controls participated in the current study. Data from two participants with aniridia were excluded from analyses (due to a significant dropout artifact and missing data). One control subject was excluded due to an anatomical abnormality, and two others were excluded because they did not match the demographic profile of an individual in the aniridia group. This left 12 individuals with aniridia ( 7 females, mean age $=36$ years, $S D=15$ ) and 12 age- and gendermatched controls ( 7 females, mean age $=35$ years, $\mathrm{SD}=14$ ) who were included in the analyses. Controls were recruited through flyers posted in the community. Participants with aniridia were recruited through the Aniridia Foundation International Conference held in 2011 and were clinically diagnosed with aniridia. Three of the participants with aniridia belonged to the same family; all other participants included in the analyses were unrelated. After written informed consent was obtained, $10 \mathrm{~mL}$ blood samples were collected from each participant with aniridia for genotyping. All participants then completed an MRI session where a high-resolution structural image and a task-free functional MRI scan were collected. During the functional scan, participants were instructed simply to keep their eyes closed without falling asleep. All activities were approved by the Institutional Review Board of the University of Georgia.

\section{GENOTYPING}

Buffy coat was extracted from each blood sample, and genomic DNA (gDNA) was isolated and aliquoted in EDTA storage tubes. Each participant's gDNA was amplified and all coding and noncoding exons of the PAX6 gene (exons 1 through 13) and the alternatively spliced exon 5a were directly sequenced and analyzed. Sequence was compared to the wild-type PAX6 cDNA sequence and mutations were identified and are reported in the nomenclature system recommended by den Dunnen and Antonarakis (2001). Eight of the 12 participants with aniridia in the current study have confirmed heterozygous loss-of-function mutations within the coding region of the PAX6 gene. The remaining four individuals had chromosomal rearrangements that are expected to abolish PAX6 expression from the variant allele, but this has not been directly tested. Clinically, the eye phenotypes of these four individuals were indistinguishable from those with confirmed nonsense mutations. The demographic and genotypic information of each participant with aniridia is provided in Table 1.

\section{IMAGING PARAMETERS}

All data were collected on a 3T GE Signa MRI (General Electric, Milwaukee, WI, USA) at the University of Georgia's Bio-Imaging Research Center. For the high resolution structural scan, images were acquired with a T1-weighted 3D FSPGR sequence (echo time $(\mathrm{TE})=$ min full, flip angle $=20^{\circ}$, field of view (FOV) $=240 \mathrm{~mm}$ $\times 240 \mathrm{~mm}$, matrix size $=256 \times 256,150$ axial slices, in-slice resolution $=0.94 \times 0.94 \mathrm{~mm}$, slice thickness $=1.2 \mathrm{~mm}$ ). For the 
Table 1 | Demographic information on all 12 participants with aniridia

\begin{tabular}{lllll}
\hline Subject & Age & Gender & Handedness & $\begin{array}{l}\text { Confirmed mutation } \\
\text { in PAX6 }\end{array}$ \\
\hline 1 & 18 & Male & Right & No \\
2 & 19 & Female & Ambidextrous & Yes (c.771delG) \\
$3^{*}$ & 20 & Male & Right & Yes (c.204delC) \\
$4^{*}$ & 24 & Female & Left & Yes (c.204delC) \\
5 & 25 & Female & Left & No \\
6 & 28 & Female & Right & Yes (c.28C $>$ T) \\
7 & 39 & Male & Right & Yes (c.481delG) \\
8 & 47 & Male & Right & No \\
$9^{*}$ & 47 & Male & Left & Yes (c.204delC) \\
10 & 51 & Female & Right & Yes (c.766-3C $>$ G) \\
11 & 53 & Female & Right & No \\
12 & 60 & Female & Ambidextrous & Yes (c.799A $>$ T) \\
\hline
\end{tabular}

*Indicates individuals from a single family.

task-free functional scan, a $\mathrm{T} 2{ }^{*}$-weighted gradient echo wholebrain EPI sequence was acquired (repetition time $(\mathrm{TR})=5000 \mathrm{~ms}$, $\mathrm{TE}=25 \mathrm{~ms}$, flip angle $=90^{\circ}, \mathrm{FOV}=256 \mathrm{~mm} \times 256 \mathrm{~mm}$, matrix size $=128 \times 128,55$ oblique slices, in-slice resolution $=2 \times 2 \mathrm{~mm}$, slice thickness $=2.4 \mathrm{~mm}, 105$ volumes, total scan time $=8 \mathrm{~min} 45 \mathrm{~s}$ ). A TR of $5000 \mathrm{~ms}$ was determined to be necessary to collect 55 slices per volume with wholebrain coverage, while being short enough to capture the lowfrequency fluctuations of the signal of interest. The first four volumes were discarded to allow for scanner stabilization. The functional scan was collected with oblique slices aligned to a plane containing the superior margin of the anterior commissure and inferior margin of the posterior commissure for each participant.

\section{DATA ANALYSIS}

Functional MRI analyses were conducted using a procedure previously implemented in our laboratory (Krafft et al., 2014) utilizing FMRIB Software Libraries (FSL, version 5.0.1; Oxford, United Kingdom) and Analysis of Functional NeuroImages (AFNI; Cox, 1996) software packages. The following preprocessing was applied in FSL for each participant: motion correction using MCFLIRT (Jenkinson et al., 2002), non-brain removal using BET (Smith, 2002), spatial smoothing (full-width at half-maximum $[\mathrm{FWHM}]=4 \mathrm{~mm}$ ), grand-mean intensity normalization, and high-pass temporal filtering $(0.01 \mathrm{~Hz})$. Registration was carried out using FLIRT (Jenkinson and Smith, 2001; Jenkinson et al., 2002), with which each functional run was aligned to the individual's structural image, transformed into MNI152 standardized space, and resampled to $2 \mathrm{~mm}$ isotropic voxels. Probabilistic independent components analysis (ICA; Beckmann and Smith, 2004) was conducted in FSL's MELODIC for each participant to denoise individual data. Components that represented noise were selected by spatial and temporal characteristics as detailed by Kelly et al. (2010) (including head motion, physiological noise, or scanner artifacts) and removed.

Between-subjects analysis was carried out using a dual regression approach that allows for voxel-wise comparisons of intrinsic functional connectivity (Beckmann et al., 2009; Filippini et al., 2009; Zuo et al., 2010). Briefly, this approach first identifies patterns of brain activity common to all participants and subsequently finds the activity on the individual level associated with that common network. This then allows for group comparisons to be made throughout the whole brain on the strength and extent of functional correlation with the main network. To prepare the data for the dual regression, individuals' preprocessed data were temporally concatenated across subjects to create a single $4 \mathrm{D}$ (three spatial dimensions $\times$ time) dataset. The concatenated dataset was decomposed using group ICA to identify large-scale patterns of functional synchrony in the entire sample of 24 participants. Forty spatially-independent components were identified using automatic dimensionality estimation. Components of interest were selected by identifying the component with the greatest spatial correlation (using FSL's "fslcc" command) with previously identified ICNs from the publicly available dataset of Beckmann et al. (2005) and visually confirmed (e.g., Smith et al., 2009; Kiviniemi et al., 2011; Ray et al., 2013).

Dual regression in FSL then was used to identify, within each subject's fMRI dataset, subject-specific temporal dynamics and associated spatial maps for each group component. This technique involved two steps. First, the full set of 40 group-ICA spatial maps (derived from both aniridia and control participants' data) was used in a linear model fit (spatial regression) against each individual's "denoised" fMRI dataset. This resulted in a matrix for each participant describing that individual's temporal dynamics for each ICA component. In the second step, each of the individuals' time courses from the first step was normalized to unit variance and used in a linear model fit (temporal regression) against their own fMRI dataset to estimate the participant's spatial maps for each component. These subject-specific whole-brain spatial maps reflected the degree to which each voxel was functionally correlated with the grouplevel network, and were transformed into $Z$-maps for group comparison. A two-sample $t$-test was then performed between the aniridia and control groups on the individual $Z$-maps corresponding to the three group components of interest. The statistical maps from these $t$-tests were thresholded at a voxelwise uncorrected $p<0.005$. To protect against false positives, a thresholding method in AFNI derived from Monte Carlo simulations (accounting for the smoothness of the data) then was applied to the statistical maps (Ward, 2000). Based on these simulations, a family-wise alpha of 0.05 was preserved with three-dimensional clusters with a minimum volume of 55 voxels.

\section{RESULTS}

Using ICA and dual regression, this study examined differences in intrinsic functional connectivity networks between individuals with aniridia and healthy controls. Groups did not differ in the amount of head motion during the scans: aniridia participants moved an average of $0.52 \mathrm{~mm}(\mathrm{SD}=0.31)$ and control participants moved $0.67 \mathrm{~mm}(\mathrm{SD}=0.76), t_{(22)}=-0.61$, $p>0.05$. The two groups also did not differ in the percent of components removed as "noise" during individual ICA $($ aniridia $=49 \%(S D=13)$; control $=41 \%(S D=11)$, $\left.t_{(22)}=1.60, p>0.05\right)$. Of the 40 ICA components identified 
in the group analysis of all 24 participants, three were selected that represented networks of interest based on visual inspection and spatial correlation with previously identified networks (Beckmann et al., 2005). These three components corresponded to the executive control network, the primary visual network, and the default mode network (Figure 1). These components correlated with Beckmann et al. (2005) ICNs with Pearson correlation values of 0.50, 0.76, and 0.64, respectively.

The analysis for each of the three ICNs showed the same pattern: participants with aniridia exhibited regions of greater functional connectivity than control participants, with no instances of controls showing greater connectivity than the aniridia group. The between group $t$-tests on the executive control network revealed multiple regions where the aniridia participants had greater functional connectivity with the network: the left middle occipital gyrus, left lingual gyrus, right culmen/dentate of the cerebellum, and right precuneus. For the primary visual network, the aniridia group showed greater connectivity in left fusiform gyrus, and for the default mode network the aniridia group had greater connectivity with right postcentral gyrus, left parahippocampal gyrus, right medial frontal gyrus, left middle frontal gyrus and left inferior frontal gyrus (Figure 2). Table 2 shows the location and size of the regions that differed between the groups for all three networks, with each cluster surpassing the $p<0.05$ family-wise error rate threshold.

\section{DISCUSSION}

This study investigated intrinsic functional connectivity during task-free fMRI in individuals with aniridia compared to healthy age- and gender-matched controls. All participants in the aniridia group had a confirmed clinical diagnosis of aniridia and 8 out of 12 had confirmed heterozygous loss-of-function mutations within the coding region of the PAX6 gene. Using an ICA and dual-regression approach, common ICNs were identified in all participants and groups were contrasted in whole-brain activity correlated with these networks. The three ICNs of interest were the executive control network, the primary visual network, and the default mode network. In all network comparisons the aniridia group showed regions of increased functional connectivity relative to controls; there were no regions in which the control subjects had stronger functional connectivity.

In the frontal-cortex-based executive control network, the aniridia group showed greater connectivity than control subjects in a number of occipital cortical regions. The recruitment of posterior visual regions to this anterior network may represent

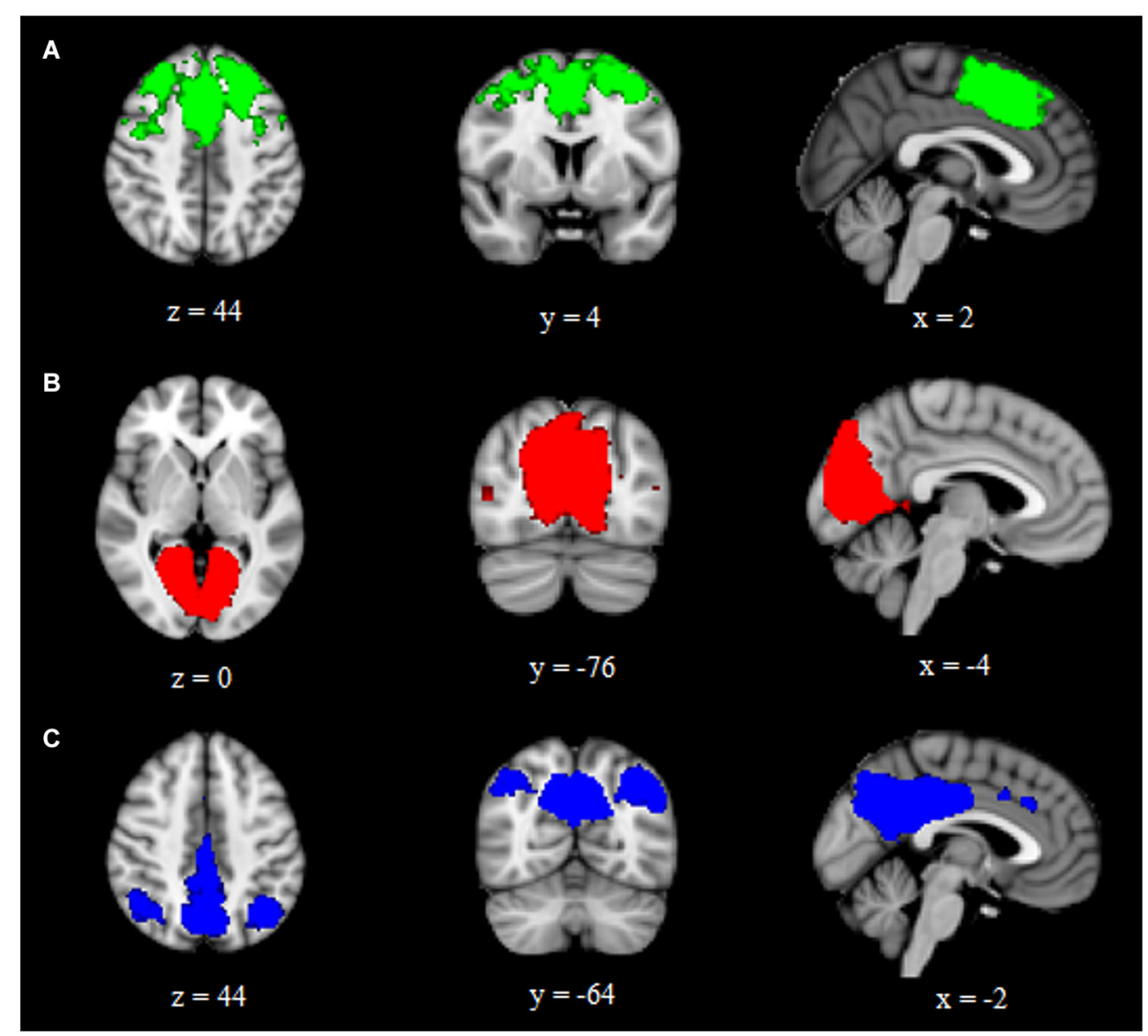

FIGURE 1 | Group ICA components representing (A) executive control, (B) primary visual, and (C) default mode intrinsic connectivity networks. Images are overlaid on the MNI152 brain and are presented in radiological orientation (right is left). 


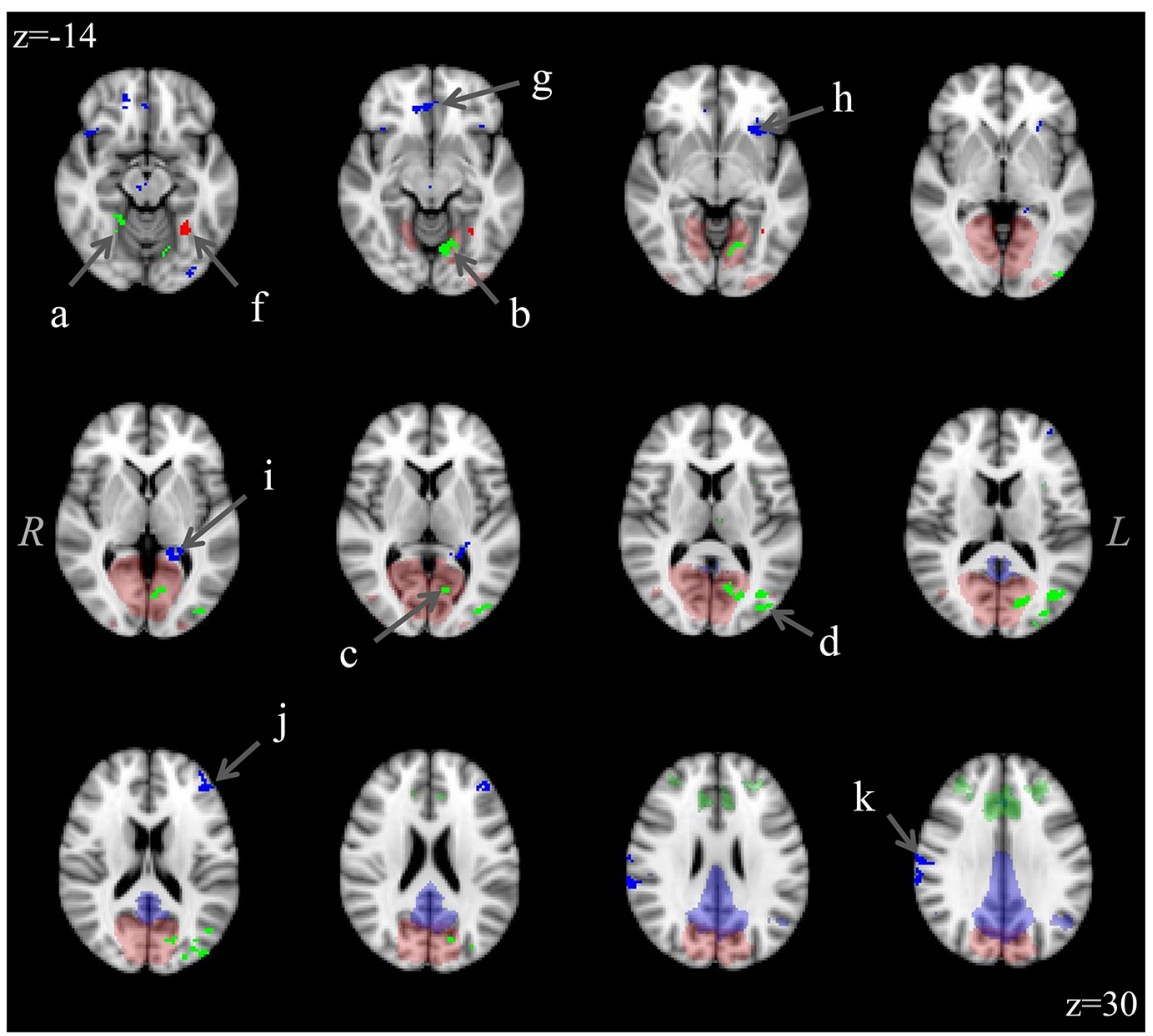

FIGURE 2 | Differences in functional connectivity within the three ICNs (executive control: green; visual: red; default mode: blue) showing greater connectivity in the aniridia group compared to controls. Difference clusters are shown in bright, opaque hues in the foreground while group ICNs are shown in transparent hues on the anatomical background image. The executive control network clusters were located in: (a) right culmen/dentate, (b) left lingual gyrus, (c) left cuneus, (d) left middle occipital gyrus; and (e) right precuneus (not shown); the visual network cluster was located in (f) left fusiform gyrus; and the default mode network clusters were located in: (g) right medial frontal gyrus, (h) left inferior frontal gyrus, (i) left parahippocampal gyrus, (j) left middle frontal gyrus; and ( $k$ ) right postcentral gyrus. T-maps were thresholded to a voxel-wise $p<0.005$ and clustered to ensure a family-wise $p<0.05$. The control group showed no regions of greater connectivity. Images are overlaid on the MNI152 brain and are presented in radiological orientation (right is left).

Table 2 | Location of significant connectivity differences.

\begin{tabular}{|c|c|c|c|}
\hline Intrinsic connectivity network & $\begin{array}{l}\text { Anatomical location } \\
\text { of cluster }\end{array}$ & $\begin{array}{l}\text { Peak } t \text {-statistic MNI } \\
\text { coordinates }(x, y, z)\end{array}$ & $\begin{array}{l}\text { Size } \\
\text { (voxels) }\end{array}$ \\
\hline \multirow[t]{5}{*}{ Executive control } & Left middle occipital gyrus & $-40,-86,2$ & 223 \\
\hline & Left cuneus & $-10,-70,2$ & 124 \\
\hline & Right culmen/dentate & $18,-52,-32$ & 101 \\
\hline & Left lingual gyrus & $-10,-70,-10$ & 81 \\
\hline & Right precuneus & $20,-8,48$ & 71 \\
\hline Primary visual & Left fusiform gyrus & $-28,-52,-16$ & 56 \\
\hline & Right medial frontal & $18,38,-18$ & 74 \\
\hline & Left middle frontal gyrus & $-40,50,16$ & 71 \\
\hline & Left inferior frontal gyrus & $-32,20,-6$ & 64 \\
\hline
\end{tabular}

MNI coordinates of the peak t-statistic and the voxel count of each cluster where the aniridia group showed greater connectivity than the control group with each of the three intrinsic connectivity networks of interest. Voxels were $2 \mathrm{~mm}^{3}$ in size and clusters had to contain at least 55 voxels to meet the criteria for significance using a family-wise error rate of $p<0.05$.

a need to supplement insufficient frontal function, as observed in the only previous fMRI study on individuals with aniridia (Ellison-Wright et al., 2004), or a greater degree of involvement of executive control in visual processing. Prefrontal cortex is involved in top-down modulation of visual processing based on current goals (Miller and Cohen, 2001; Gazzaley et al., 2007), and 
the increased connectivity between these regions in the aniridia group could be related to an increased demand for executive control due to altered visual input.

A similar pattern was observed in the analysis of the primary visual network: participants in the aniridia group showed greater intrinsic connectivity than controls in left fusiform gyrus, a region which is not typically part of this network in healthy individuals. This suggests that individuals with aniridia are utilizing proximal cortical regions to supplement the functioning of the hub of this network, possibly due to a lack of efficiency within the network proper. This inefficiency may be caused directly by structural abnormalities related to altered PAX6 gene function (Free et al., 2003; Ellison-Wright et al., 2004) or by the ocular deficits associated with aniridia (e.g., poor visual acuity, cataract, glaucoma, foveal hypoplasia) which necessarily reduce the efficacy of functional input to the occipital lobe.

Finally, in the default mode network, some of the regions (medial temporal and frontal) that showed increased functional connectivity were similar to those reported by Ellison-Wright et al. (2004) to have altered structure or task activation in a single family with aniridia. Hippocampal and medial frontal regions have been noted in some studies to be part of the normal default mode network (Vincent et al., 2006; Buckner et al., 2008), and the increased connectivity in nearby parahippocampal and inferior and lateral frontal cortex in the current study suggests that the functioning within the default mode network in aniridia was reduced compared to controls such that these adjacent regions were recruited.

When considering the functional connectivity across all three ICNs, it is evident that the regions where the aniridia group exhibited greater connectivity than controls were primarily located within occipital and frontal cortex, as well as the medial temporal lobe. The occipital lobe constitutes the core of visual processing in the brain, which is the sensory modality most notably disrupted by heterozygous loss-of-function mutations of the PAX6 gene. Furthermore, reductions in gray matter concentration in the occipital lobe have been identified previously in individuals with aniridia (Free et al., 2003). White matter deficits in occipital cortex and gray matter volume excesses in frontal and medial temporal lobes also have been reported in individuals with aniridia, in addition to reduced task-based activation of frontal regions during a verbal executive function paradigm (EllisonWright et al., 2004). These differences suggest that PAX6 plays a particularly active role in the development of these regions and structural abnormalities may lead to these regions being recruited to a greater extent during task-free activity in order to bolster the efficiency of the network, potentially allowing for better functioning during active behaviors (Sharp et al., 2011).

Individuals with PAX6-mediated aniridia have insufficient levels of PAX6 protein, which is expressed in the eye, cerebellum, olfactory bulb and throughout the cerebral cortex in early human brain development, but is not expressed cortically in the adult (Walther and Gruss, 1991; Stoykova and Gruss, 1994; Georgala et al., 2011). The differences in functional connectivity demonstrated in this study likely represent complex and compounding effects of PAX6 haploinsufficiency. These aberrant patterns of activity in the adult could be a product of: (1) inefficient local neural circuits, as a direct consequence of functional PAX6 reduction or through putative genomic targets involved in connectivity during early cortical development; (2) irregular connectivity due to the underdevelopment of visual and olfactory sensory input structures; (3) cortical reorganization in late-stage brain development due to a lack of or atypical environmental sensory input; or (4) combined effects of the above. The results of the current study reflect differences in large-scale functional network dynamics; given that PAX6 has an extensive role in early neural development and has noteworthy anatomical and functional effects on sensory structures, the findings of the current study are ostensibly due to an amalgam of direct molecular effects of PAX6 in early development and indirect consequences of these effects occurring later in the lifespan once PAX6 is no longer expressed.

In regards to the present findings, there are a few potential sources of error that should be considered. First, structural abnormalities were not measured in the current sample and, thus, conclusions about the relationship between structure and function were based on previously reported structural differences in several brain regions in aniridia (Free et al., 2003; Ellison-Wright et al., 2004). As such, one cannot exclude the possibility that disrupted local gray matter structure or altered structural connectivity in the current aniridia sample contributed to the observed differences in functional connectivity in an undetermined manner. Additionally, in all of the group ICN comparisons, the aniridia group exhibited regions of increased connectivity whereas the control group exhibited no such regions. This leads to the question of whether increased connectivity or diffuse functional networks are a non-specific feature of intrinsic functional neural organization in aniridia. Further research is required to investigate this possibility in other individuals with aniridia using alternative analytical approaches. It also may be fruitful to explore the developmental trajectory of these connectivity patterns in aniridia to clarify the interaction of functional experience with structural changes in formation of these networks.

\section{CONCLUSIONS}

This study investigated intrinsic functional connectivity in individuals with aniridia compared to healthy controls. Increased connectivity was observed in the aniridia group in three ICNs: the executive control, primary visual, and default mode networks. Specifically, individuals with aniridia recruited supplementary occipital, frontal, and medial temporal regions not identified in matched controls, suggesting a lack of network specificity and refinement related to mutations of the PAX6 gene. Though the specific causes of these functional connectivity differences remain undetermined at present, mutations in the PAX6 gene evidently do interfere with the formation of discrete cognitive networks and necessitate greater functional involvement of additional neural regions. Further research is required to investigate how these unique patterns of functional co-activations in aniridia derive from neurophysiological mechanisms of PAX6 at the cellular and molecular level, as well as how they may contribute to behavioral differences. 


\section{ACKNOWLEDGMENTS}

This research was supported by the Sharon Stewart Aniridia Research Trust and the University of Georgia Bio-Imaging Research Center.

\section{REFERENCES}

Bamiou, D. E., Free, S. L., Sisodiya, S. M., Chong, W. K., Musiek, F., Williamson, K. A., et al. (2007). Auditory interhemispheric transfer deficits, hearing difficulties and brain magnetic resonance imaging abnormalities in children with congenital aniridia due to PAX6 mutations. Arch. Pediatr. Adolesc. Med. 161, 463-469. doi: 10.1001/archpedi.161.5.463

Bamiou, D. E., Musiek, F. E., Sisodiya, S. M., Free, S. L., Davies, R. A., Moore, A., et al. (2004). Deficient auditory interhemispheric transfer in patients with PAX6 mutations. Ann. Neurol. 56, 503-509. doi: 10.1002/ana.20227

Beckmann, C. F., DeLuca, M., Devlin, J. T., and Smith, S. M. (2005). Investigations into resting-state connectivity using independent component analysis. Philos. Trans. R. Soc. Lond. B Biol. Sci. 360, 1001-1013. doi: 10.1098/rstb.2005.1634

Beckmann, C. F., Mackay, C. E., Filippini, N., and Smith, S. M. (2009). "Group comparison of resting-state FMRI data using multi-subject ICA and dual regression," in Presented at Organization for Human Brain Mapping (OHBM) 15th Annual Meeting. San Francisco, CA: USA.

Beckmann, C. F., and Smith, S. M. (2004). Probabilistic independent component analysis for functional magnetic resonance imaging. IEEE Trans. Med. Imaging 23, 137-152. doi: 10.1109/tmi.2003.822821

Biswal, B., Yetkin, F. Z., Haughton, V. M., and Hyde, J. S. (1995). Functional connectivity in the motor cortex of resting human brain using echo-planar MRI. Magn. Reson. Med. 34, 537-541. doi: 10.1002/mrm.1910340409

Boretius, S., Michaelis, T., Tammer, R., Ashery-Padan, R., Frahm, J., and Stoykova, A. (2009). In vivo MRI of altered brain anatomy and fiber connectivity in adult pax6 deficient mice. Cereb. Cortex 19, 2838-2847. doi: 10.1093/cercor/bhp057

Buckner, R. L., Andrews-Hanna, J. R., and Schacter, D. L. (2008). The brain's default network: anatomy, function and relevance to disease. Ann. N Y Acad. Sci. 1124, 1-38. doi: 10.1196/annals.1440.011

Cox, R. W. (1996). AFNI: software for analysis and visualization of functional magnetic resonance neuroimages. Comput. Biomed. Res. 29, 162-173. doi: 10. 1006/cbmr.1996.0014

Damoiseaux, J. S., Rombouts, S. A., Barkhof, F., Scheltens, P., Stam, C. J., Smith, S. M., et al. (2006). Consistent resting-state networks across healthy subjects. Proc. Natl. Acad. Sci. U S A 103, 13848-13853. doi: 10.1073/pnas.0601417103

De Luca, M., Beckmann, C. F., De Stefano, N., Matthews, P. M., and Smith, S. M. (2006). fMRI resting state networks define distinct modes of long-distance interactions in the human brain. Neuroimage 29, 1359-1367. doi: 10.1016/j. neuroimage.2005.08.035

den Dunnen, J. T., and Antonarakis, S. E. (2001). Nomenclature for the description of human sequence variations. Hum. Genet. 109, 121-124. doi: 10. 1007/s004390100505

Ellison-Wright, Z., Heyman, I., Frampton, I., Rubia, K., Chitnis, X., Ellison-Wright, I., et al. (2004). Heterozygous PAX6 mutation, adult brain structure and frontostriato-thalamic function in a human family. Eur. J. Neurosci. 19, 1505-1512. doi: 10.1111/j.1460-9568.2004.03236.x

Estivill-Torrus, G., Pearson, H., van Heyningen, V., Price, D. J., and Rashbass, P. (2002). Pax6 is required to regulate the cell cycle and the rate of progression from symmetrical to asymmetrical division in mammalian cortical progenitors. Development 129, 455-466.

Fantes, J., Redeker, B., Breen, M., Boyle, S., Brown, J., Fletcher, J., et al. (1995). Aniridia-associated cytogenetic rearrangements suggest that a position effect may cause the mutant phenotype. Hum. Mol. Genet. 4, 415-422. doi: 10. 1093/hmg/4.3.415

Filippini, N., MacIntosh, B. J., Hough, M. G., Goodwin, G. M., Frisoni, G. B., Smith, S. M., et al. (2009). Distinct patterns of brain activity in young carriers of the APOE-epsilon4 allele. Proc. Natl. Acad. Sci. U S A 106, 7209-7214. doi: 10. 1073/pnas.0811879106

Fischbach, B. V., Trout, K. L., Lewis, J., Luis, C. A., and Sika, M. (2005). WAGR syndrome: a clinical review of 54 cases. Pediatrics 116, 984-988. doi: 10. 1542/peds.2004-0467

Fox, M. D., and Raichle, M. E. (2007). Spontaneous fluctuations in brain activity observed with functional magnetic resonance imaging. Nat. Rev. Neurosci. 8, 700-711. doi: 10.1038/nrn2201
Fox, M. D., Snyder, A. Z., Vincent, J. L., Corbetta, M., Van Essen, D. C., and Raichle, M. E. (2005). The human brain is intrinsically organized into dynamic, anticorrelated functional networks. Proc. Natl. Acad. Sci. U S A 102, 9673-9678. doi: 10.1073/pnas.0504136102

Fransson, P. (2005). Spontaneous low-frequency BOLD signal fluctuations: an fMRI investigation of the resting-state default mode of brain function hypothesis. Hum. Brain Mapp. 26, 15-29. doi: 10.1002/hbm.20113

Free, S. L., Mitchell, T. N., Williamson, K. A., Churchill, A. J., Shorvon, S. D., Moore, A. T., et al. (2003). Quantitative MR image analysis in subjects with defects in the PAX6 gene. Neuroimage 20, 2281-2290. doi: 10.1016/j.neuroimage.2003.07. 001

Gazzaley, A., Rissman, J., Cooney, J., Rutman, A., Seibert, T., Clapp, W., et al. (2007). Functional interactions between prefrontal and visual association cortex contribute to top-down modulation of visual processing. Cereb. Cortex 17(Suppl. 1), i125-i135. doi: 10.1093/cercor/bhm113

Gehring, W. J. (1996). The master control gene for morphogenesis and evolution of the eye. Genes Cells 1, 11-15. doi: 10.1046/j.1365-2443.1996.11011.x

Georgala, P. A., Carr, C. B., and Price, D. J. (2011). The role of Pax6 in forebrain development. Dev. Neurobiol. 71, 690-709. doi: 10.1002/dneu.20895

Götz, M., Stoykova, A., and Gruss, P. (1998). Pax6 controls radial glia differentiation in the cerebral cortex. Neuron 21, 1031-1044. doi: 10.1016/s08966273(00)80621-2

Gramer, E., Reiter, C., and Gramer, G. (2012). Glaucoma and frequency of ocular and general diseases in 30 patients with aniridia: a clinical study. Eur. J. Ophthalmol. 22, 104-110. doi: 10.5301/EJO.2011.8318

Greicius, M. D., Krasnow, B., Reiss, A. L., and Menon, V. (2003). Functional connectivity in the resting brain: a network analysis of the default mode hypothesis. Proc. Natl. Acad. Sci. U S A 100, 253-258. doi: 10.1073/pnas.0135058100

Gusnard, D. A., Akbudak, E., Shulman, G. L., and Raichle, M. E. (2001). Medial prefrontal cortex and self-referential mental activity: relation to a default mode of brain function. Proc. Natl. Acad. Sci. U S A 98, 4259-4264. doi: 10.1073/pnas. 071043098

Halder, G., Callaerts, P., and Gehring, W. J. (1995). New perspectives on eye evolution. Curr. Opin. Genet. Dev. 5, 602-609. doi: 10.1016/0959-437x(95)80029-8

Heins, N., Malatesta, P., Cecconi, F., Nakafuku, M., Tucker, K. L., Hack, M. A., et al. (2002). Glial cells generate neurons: the role of the transcription factor Pax6. Nat. Neurosci. 5, 308-315. doi: 10.1038/nn828

Heyman, I., Frampton, I., van Heyningen, V., Hanson, I., Teague, P., Taylor, A., et al. (1999). Psychiatric disorder and cognitive function in a family with an inherited novel mutation of the developmental control gene PAX6. Psychiatr. Genet. 9, 85-90. doi: 10.1097/00041444-199906000-00006

Hingorani, M., Hanson, I., and van Heyningen, V. (2012). Aniridia. Eur. J. Hum. Genet. 20, 1011-1017. doi: 10.1038/ejhg.2012.100

Hingorani, M., Williamson, K. A., Moore, A. T., and van Heyningen, V. (2009). Detailed ophthalmologic evaluation of 43 individuals with PAX6 mutations. Invest. Ophthalmol. Vis. Sci. 50, 2581-2590. doi: 10.1167/iovs.08-2827

Jenkinson, M., Bannister, P., Brady, M., and Smith, S. (2002). Improved optimization for the robust and accurate linear registration and motion correction of brain images. Neuroimage 17, 825-841. doi: 10.1006/nimg.2002.1132

Jenkinson, M., and Smith, S. (2001). A global optimisation method for robust affine registration of brain images. Med. Image Anal. 5, 143-156. doi: 10.1016/s13618415(01)00036-6

Kannurpatti, S. S., Rypma, B., and Biswal, B. B. (2012). Prediction of taskrelated BOLD fMRI with amplitude signatures of resting-state fMRI. Front. Syst. Neurosci. 6:7. doi: 10.3389/fnsys.2012.00007

Kelly, R. E. Jr., Alexopoulos, G. S., Wang, Z., Gunning, F. M., Murphy, C. F., Morimoto, S. S., et al. (2010). Visual inspection of independent components: defining a procedure for artifact removal from fMRI data. J. Neurosci. Methods 189, 233-245. doi: 10.1016/j.jneumeth.2010.03.028

Kim, H. (2010). Dissociating the roles of the default-mode, dorsal and ventral networks in episodic memory retrieval. Neuroimage 50, 1648-1657. doi: 10. 1016/j.neuroimage.2010.01.051

Kiviniemi, V., Vire, T., Remes, J., Elseoud, A. A., Starck, T., Tervonen, O., et al. (2011). A sliding time-window ICA reveals spatial variability of the default mode network in time. Brain Connect. 1, 339-347. doi: 10.1089/brain.2011.0036

Krafft, C. E., Pierce, J. E., Schwarz, N. F., Chi, L., Weinberger, A. L., Schaeffer, D. J., et al. (2014). An eight month randomized controlled exercise intervention alters resting state synchrony in overweight children. Neuroscience 256, 445-455. doi: 10.1016/j.neuroscience.2013.09.052 
Lauderdale, J. D., Wilensky, J. S., Oliver, E. R., Walton, D. S., and Glaser, T. (2000). $3^{\prime}$ deletions cause aniridia by preventing PAX6 gene expression. Proc. Natl. Acad. Sci. U S A 97, 13755-13759. doi: 10.1073/pnas.240398797

Lee, H., Khan, R., and O'Keefe, M. (2008). Aniridia: current pathology and management. Acta Ophthalmol. 86, 708-715. doi: 10.1111/j.1755-3768.2008. 01427.x

Liu, Y., Yu, C., Liang, M., Li, J., Tian, L., Zhou, Y., et al. (2007). Whole brain functional connectivity in the early blind. Brain 130, 2085-2096. doi: 10. 1093/brain/awm121

McCulley, T. J., Mayer, K., Dahr, S. S., Simpson, J., and Holland, E. J. (2005). Aniridia and optic nerve hypoplasia. Eye 19, 762-764. doi: 10.1038/sj.eye. 6701642

Miller, E. K., and Cohen, J. D. (2001). An integrative theory of prefrontal cortex function. Annu. Rev. Neurosci. 24, 167-202. doi: 10.1146/annurev.neuro. 24.1.167

Mitchell, T. N., Free, S. L., Williamson, K. A., Stevens, J. M., Churchill, A. J., Hanson, I. M., et al. (2003). Polymicrogyria and absence of pineal gland due to PAX6 mutation. Ann. Neurol. 53, 658-663. doi: 10.1002/ana.10576

Nelson, L. B., Spaeth, G. L., Nowinski, T. S., Margo, C. E., and Jackson, L. (1984). Aniridia. A review. Surv. Ophthalmol. 28, 621-642. doi: 10.1016/00396257(84)90184-x

Osumi, N., Shinohara, H., Numayama-Tsuruta, K., and Maekawa, M. (2008). Concise review: Pax6 transcription factor contributes to both embryonic and adult neurogenesis as a multifunctional regulator. Stem Cells 26, 1663-1672. doi: 10.1634/stemcells.2007-0884

Qin, W., Liu, Y., Jiang, T., and Yu, C. (2013). The development of visual areas depends differently on visual experience. PLoS One 8:e53784. doi: 10. 1371/journal.pone.0053784

Raichle, M. E., MacLeod, A. M., Snyder, A. Z., Powers, W. J., Gusnard, D. A., and Shulman, G. L. (2001). A default mode of brain function. Proc. Natl. Acad. Sci. U S A 98, 676-682. doi: 10.1073/pnas.98.2.676

Ray, K. L., McKay, D. R., Fox, P. M., Riedel, M. C., Uecker, A. M., Beckmann, C. F., et al. (2013). ICA model order selection of task co-activation networks. Front. Neurosci. 7:237. doi: 10.3389/fnins.2013.00237

Robinson, D. O., Howarth, R. J., Williamson, K. A., van Heyningen, V., Beal, S. J., and Crolla, J. A. (2008). Genetic analysis of chromosome 11p13 and the PAX6 gene in a series of 125 cases referred with aniridia. Am. J. Med. Genet. A 146A, 558-569. doi: 10.1002/ajmg.a.32209

Rogers, B. P., Morgan, V. L., Newton, A. T., and Gore, J. C. (2007). Assessing functional connectivity in the human brain by fMRI. Magn. Reson. Imaging 25, 1347-1357. doi: 10.1016/j.mri.2007.03.007

Sala-Llonch, R., Peña-Gómez, C., Arenaza-Urquijo, E. M., Vidal-Pineiro, D., Bargallo, N., Junque, C., et al. (2012). Brain connectivity during resting state and subsequent working memory task predicts behavioural performance. Cortex 48 , 1187-1196. doi: 10.1016/j.cortex.2011.07.006

Schwartz, F., Neve, R., Eisenman, R., Gessler, M., and Bruns, G. (1994). A WAGR region gene between PAX-6 and FSHB expressed in fetal brain. Hum. Genet. 94, 658-664. doi: 10.1007/bf00206960

Seeley, W. W., Menon, V., Schatzberg, A. F., Keller, J., Glover, G. H., Kenna, H., et al. (2007). Dissociable intrinsic connectivity networks for salience processing and executive control. J. Neurosci. 27, 2349-2356. doi: 10.1523/jneurosci.5587-06. 2007

Sharp, D. J., Beckmann, C. F., Greenwood, R., Kinnunen, K. M., Bonnelle, V., De Boissezon, X., et al. (2011). Default mode network functional and structural connectivity after traumatic brain injury. Brain 134, 2233-2247. doi: 10 . 1093/brain/awr175

Sisodiya, S. M., Free, S. L., Williamson, K. A., Mitchell, T. N., Willis, C., Stevens, J. M., et al. (2001). PAX6 haploinsufficiency causes cerebral malformation and olfactory dysfunction in humans. Nat. Genet. 28, 214-216. doi: 10.1038/90042
Smith, S. M. (2002). Fast robust automated brain extraction. Hum. Brain Mapp. 17, 143-155. doi: 10.1002/hbm.10062

Smith, S. M., Fox, P. T., Miller, K. L., Glahn, D. C., Fox, P. M., Mackay, C. E., et al. (2009). Correspondence of the brain's functional architecture during activation and rest. Proc. Natl. Acad. Sci. U S A 106, 13040-13045. doi: 10.1073/pnas. 0905267106

Stoykova, A., and Gruss, P. (1994). Roles of Pax-genes in developing and adult brain as suggested by expression patterns. J. Neurosci. 14, 1395-1412.

Thompson, P. J., Mitchell, T. N., Free, S. L., Williamson, K. A., Hanson, I. M., van Heyningen, V., et al. (2004). Cognitive functioning in humans with mutations of the PAX6 gene. Neurology 62, 1216-1218. doi: 10.1212/01.wnl.0000118298. 81140.62

Ton, C. C., Hirvonen, H., Miwa, H., Weil, M. M., Monaghan, P., Jordan, T., et al. (1991). Positional cloning and characterization of a paired box- and homeoboxcontaining gene from the aniridia region. Cell 67, 1059-1074. doi: 10.1016/00928674(91)90284-6

Tuoc, T. C., Radyushkin, K., Tonchev, A. B., Piñon, M. C., Ashery-Padan, R. Molnár, Z., et al. (2009). Selective cortical layering abnormalities and behavioral deficits in cortex-specific Pax6 knock-out mice. J. Neurosci. 29, 8335-8349. doi: 10.1523/JNEUROSCI.5669-08.2009

van Heyningen, V., and Williamson, K. A. (2002). PAX6 in sensory development. Hum. Mol. Genet. 11, 1161-1167. doi: 10.1093/hmg/11.10.1161

Vincent, J. L., Snyder, A. Z., Fox, M. D., Shannon, B. J., Andrews, J. R., Raichle, M. E., et al. (2006). Coherent spontaneous activity identifies a hippocampalparietal memory network. J. Neurophysiol. 96, 3517-3531. doi: 10.1152/jn. 00048.2006

Walther, C., and Gruss, P. (1991). Pax-6, a murine paired box gene, is expressed in the developing CNS. Development 113, 1435-1449.

Ward, B. D. (2000). Simultaneous inference for fMRI data. Milwaukee, WI, USA: Biophysics Research Institute, Medical College of Wisconsin.

Warren, N., Caric, D., Pratt, T., Clausen, J. A., Asavaritikrai, P., Mason, J. O., et al. (1999). The transcription factor, Pax6, is required for cell proliferation and differentiation in the developing cerebral cortex. Cereb. Cortex 9, 627-635. doi: 10.1093/cercor/9.6.627

Yu, C., Liu, Y., Li, J., Zhou, Y., Wang, K., Tian, L., et al. (2008). Altered functional connectivity of primary visual cortex in early blindness. Hum. Brain Mapp. 29 , 533-543. doi: 10.1002/hbm.20420

Zuo, X. N., Kelly, C., Adelstein, J. S., Klein, D. F., Castellanos, F. X., and Milham, M. P. (2010). Reliable intrinsic connectivity networks: test-retest evaluation using ICA and dual regression approach. Neuroimage 49, 2163-2177. doi: 10. 1016/j.neuroimage.2009.10.080

Conflict of Interest Statement: The authors declare that the research was conducted in the absence of any commercial or financial relationships that could be construed as a potential conflict of interest.

Received: 30 May 2014; accepted: 29 November 2014; published online: 19 December 2014.

Citation: Pierce JE, Krafft CE, Rodrigue AL, Bobilev AM, Lauderdale JD and McDowell JE (2014) Increased functional connectivity in intrinsic neural networks in individuals with aniridia. Front. Hum. Neurosci. 8:1013. doi: 10.3389/fnhum.2014.01013

This article was submitted to the journal Frontiers in Human Neuroscience.

Copyright (C) 2014 Pierce, Krafft, Rodrigue, Bobilev, Lauderdale and McDowell. This is an open-access article distributed under the terms of the Creative Commons Attribution License (CC BY). The use, distribution and reproduction in other forums is permitted, provided the original author(s) or licensor are credited and that the original publication in this journal is cited, in accordance with accepted academic practice. No use, distribution or reproduction is permitted which does not comply with these terms. 